\title{
Г.Ю. Лаванов
}

Национальный авиационный университет, Киев

E-mail: Lavanovgennady@gmail.com

\section{Двойной метамагнитный фазовый переход в изинговском антиферромагнетике с легкоплоскостной одноионной анизотропией и со спином ионов $S=1$}

Представлено академиком НАН Украины В.М. Локтевым

Показано, что в изинговском АФМ со спином ионов $S=1$ магнитное поле при $T \neq 0$ может индуцировать двойной метамагнитный переход в виде последовательности двух квантовых магнитных фазовых переходов первого рода (QPT I) с плато в намагничивании между этими переходами. При этом переход из антиферромагнитной фазы в ферромагнитную происходит через промежуточное состояние, в котором спин подрешетки, исходно намагниченный против магнитного поля, находится в ван-флековском парамагнитном состоянии.

Ключевые слова: энтропия, антиферромагнетик, парамагнетик, фазовые переходы.

Исследованию магнитных свойств сильно анизотропных магнетиков уделяется большое внимание [1-3]. В них при низких температурах могут наблюдаться магнитные квантовые фазовые переходы (QPT) [2-6]. Для антиферромагнетиков такие переходы могут быть, как показано в [7], подобными метамагнитным фазовым переходам с плато в намагничивании.

Идеология QPT появилась после пионерской работы Герца [8], которая дала возможность классифицировать их как отдельный вид фазовых переходов. Одной из причин, приводящей к QPT, являются квантовые флуктуации, неизбежно следующие из принципа неопределенности Гейзенберга. В изинговской системе спинов к QPT [9] могут приводить квантовые флуктуации, связанные с конкурирующими одноионной анизотропией легкоплоскостного типа и обменными взаимодействиями.

Различают два вида QPT: первого рода, со скачкообразным изменением параметра порядка [9] и второго рода, при котором происходит непрерывное изменение параметра порядка [10].

Особый интерес вызывает QPT первого рода, реализующийся при $T \neq 0$, когда несмотря на конечную температуру и приложенное внешнее поле $(h)$ намагничивание имеет ступенчатый характер с образованием “плато” [7], в интервале полей существования которого намагниченность слабо зависит от поля.

(C) Г.Ю. Лаванов, 2018 
Целью этой работы является описание магнитных квантовых фазовых переходов при $T \neq 0$ в изинговском АФМ с положительной константой одноионной анизотропии $(D>0)$ и со спином ионов $S=1$. Будет показано, что в изинговском двухподрешеточном АФМ возможен двойной метамагнитный фазовый переход. В классических антиферромагнетиках неелевского типа двойной метамагнитный переход возможен, если количество подрешеток больше, чем две [11].

Рассмотрим гамильтониан системы:

$$
H=\frac{1}{2} \sum_{\mathbf{n}_{\alpha}, m_{\beta}} I_{\mathbf{n}_{\alpha}, \mathbf{m}_{\beta}} S_{\mathbf{n}_{\alpha}}^{z} S_{\mathbf{m}_{\beta}}^{z}+D \sum_{\mathbf{n}_{\alpha}}\left(S_{\mathbf{n}_{\alpha}}^{z}\right)^{2}-h \sum_{\mathbf{n}_{\alpha}} S_{\mathbf{n}_{\alpha}}^{z}
$$

где положение между магнитными ионами задается векторами $\mathbf{n}_{\alpha}$ и $\mathbf{m}_{\beta} ; \alpha$ и $\beta$ - нумеруют магнитные подрешетки; $S^{z}$ - оператор проекции спина. В (1) константы гамильтониана и магнитное поле заданы в энергетических единицах.

Ограничимся рассмотрением взаимодействия ближайших соседей. Тогда, обозначив константу межподрешеточного взаимодействия $(\alpha \neq \beta)$ через $I_{1}>0$, а внутриподрешеточного $(\alpha=\beta)-I_{2}$ (ее вклад в свободную энергию будем брать со знаком “минус"). При этом величина $I_{2}$ по модулю должна оставаться значительно меньшей $I_{1}$, чтобы не нарушалось приближение двухподрешеточной модели. Константы взаимодействия $I_{1}$ и $I_{2}$ могут быть выражены с учетом ближайших соседей посредством соотношений: $I_{1}=z_{\alpha \alpha} I_{\mathbf{n}_{\alpha}} \mathbf{m}_{\alpha}$ , где $z_{\alpha \alpha}-$ число ближайших соседей внутри подрешетки, а $I_{2}=z_{\mathbf{n}_{\alpha} \mathbf{m}_{\beta}} I_{\mathbf{n}_{\alpha} \mathbf{m}_{\beta}}$, где $z_{\mathbf{n}_{\alpha} \mathbf{m}_{\beta}}-$ число ближайших соседей другой подрешетки.

При $T=0$ энергия основного состояния АФМ может быть записана в виде [9]:

$$
\begin{aligned}
& E_{T=0}=I_{1}<s_{1}^{z}><s_{2}^{z}>-\frac{1}{2} I_{2}\left(<s_{1}^{z}>^{2}+<s_{2}^{z}>^{2}\right)+ \\
& +D\left(<\left(s_{1}^{z}\right)^{2}>+<\left(s_{2}^{z}\right)^{2}>\right)-h\left(<s_{1}^{z}>+<s_{2}^{z}>\right),
\end{aligned}
$$

где $<s_{1}^{z}>,<s_{2}^{z}>,<\left(s_{1}^{z}\right)^{2}>,<\left(s_{2}^{z}\right)^{2}>-$ квантовые средние спиновых состояний ионов подрешеток, которые в приближении самосогласованного поля могут принимать значения равные \pm 1 или 0 [7].

Для $T=0$, как показано в [7], минимум (2) в качестве основного дает АФМ состояние с $<s_{1}^{z}>=-<s_{2}^{z}>=1$ которое при вводе магнитного поля первым родом переходит в промежуточное состояние $<s_{1}^{z}>=1,<s_{2}^{z}>=0$. В сильных полях реализуется ФМ фаза $\mathrm{c}<s_{1}^{z}>=<s_{2}^{z}>=1$.

Для $T \neq 0$ свободная энергия в расчете на два спина магнитной ячейки может быть записана в виде:

$$
\begin{aligned}
& F=I_{1} s_{1} s_{2}-\frac{1}{2} I_{2}\left(s_{1}^{2}+s_{2}^{2}\right)+D\left(q_{z z}^{(1)}+q_{z z}^{(2)}\right)-h\left(s_{1}+s_{2}\right)+ \\
& +T\left(\frac{q_{z z}^{(1)}+S_{1}}{2} \ln \frac{q_{z z}^{(1)}+s_{1}}{2}+\frac{q_{z z}^{(1)}-s_{1}}{2} \ln \frac{q_{z z}^{(1)}-s_{1}}{2}+\right. \\
& +\frac{q_{z z}^{(2)}+S_{2}}{2} \ln \frac{q_{z z}^{(2)}+S_{2}}{2}+\frac{q_{z z}^{(2)}-S_{2}}{2} \ln \frac{q_{z z}^{(2)}-S_{2}}{2}+\left(1-q_{z z}^{(1)}\right) \ln \left(1-q_{z z}^{(1)}\right)+\left(1-q_{z z}^{(2)}\right),
\end{aligned}
$$




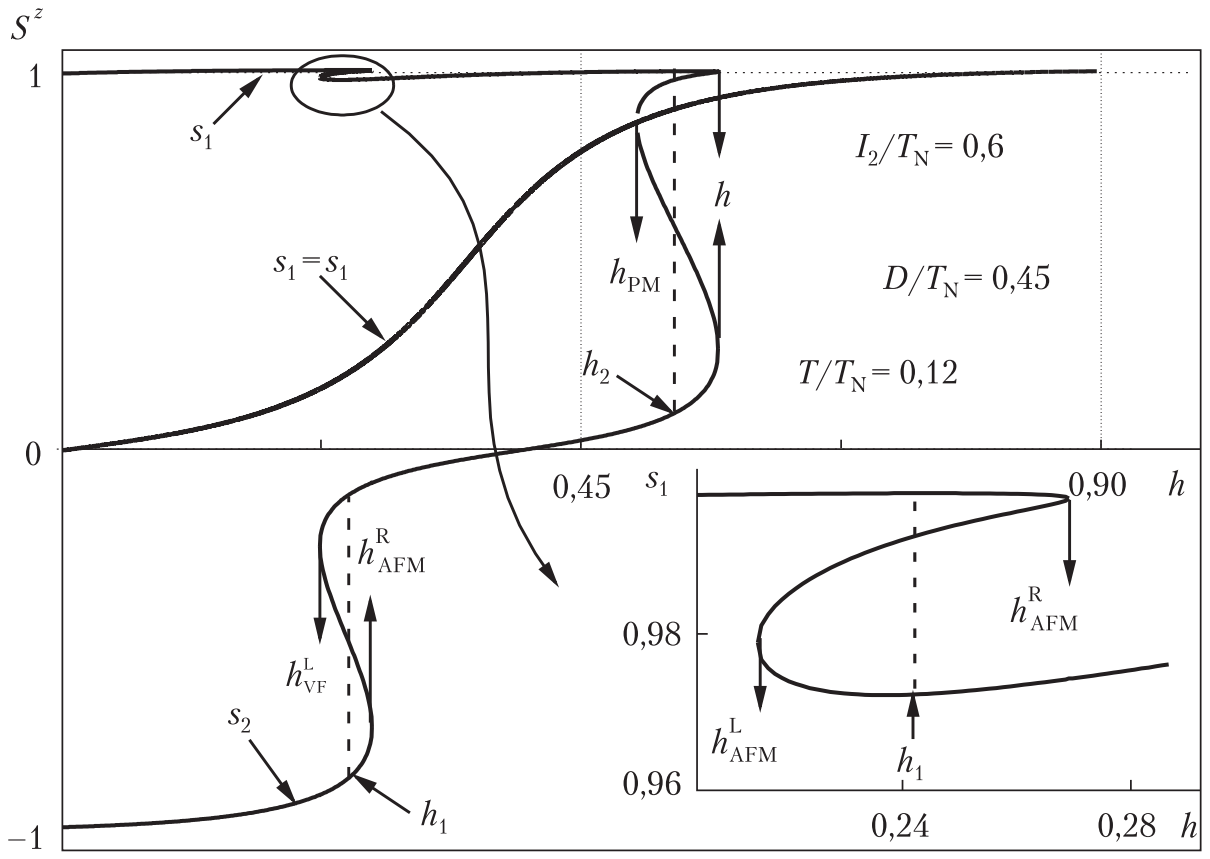

Puc. 1. Полевая зависимость для спинов $s_{1}(h)$ и $s_{1}(h)$ при $D / T_{\mathrm{N}}=0,45$ и $T / T_{\mathrm{N}}=0,12$. Константы обмена $I_{2} / T_{\mathrm{N}}=0,6$, где $T_{\mathrm{N}}=I_{1}+I_{2}$. Стрелками показаны границы потери устойчивости решений уравнения состояния, штриховые линии - точки равновесного QPT. На вставке приведена зависимость $s_{1}(h)$ вблизи QPT

где $s_{1}, s_{2}, q_{z z}^{(1)}$ и $q_{z z}^{(2)}$ - термодинамические средние $z$-проекций спинов и $z z$-компонент спиновых квадрупольных моментов подрешеток.

Для поиска равновесных состояний будем пользоваться уравнениями состояния:

$$
\frac{\partial F}{\partial q_{z z}^{(1)}}=0 ; \quad \frac{\partial F}{\partial s_{1}}=0 ; \quad \frac{\partial F}{\partial q_{z z}^{(2)}}=0 ; \quad \frac{\partial F}{\partial s_{2}}=0 .
$$

Решения уравнений (4) модели (3) позволяют получить полевые зависимости для средних значений спина подрешеток при $T \neq 0$.

Для примера на рис. 1 показаны полевые зависимости проекций спинов подрешеток, полученные из уравнений состояния для температуры $T / T_{\mathrm{N}}=0,12$. Как видно из рисунка, численное решение уравнения состояния дает следующие решения.

1. Антиферромагнитное (АФ) состояние. В отсутствии внешнего магнитного поля $(h=0)$, устойчивым является АФ состояние $S_{1}(\tau, h=0)=-S_{2}(\tau, h=0)$. При возрастании величины магнитного поля величина среднего спина $s_{1}$ первой подрешетки, ориентированной вдоль поля, изменяется слабо, оставаясь почти все время близкой к насыщению. Более существенно изменяется величина среднего спина второй подрешетки $s_{2}\left(s_{2}<0\right)$, который изначально направлен против внешнего поля.

В силу сильной нелинейности модели, по мере роста внешнего поля $h$ появляется неустойчивое решение с $\frac{\partial s_{1}}{\partial h}<0, \frac{\partial s_{2}}{\partial h}<0$ в интервале $\left[h_{\mathrm{VF}}^{L}, h_{\mathrm{AFM}}\right]$, границы которого на рис. 1 
Puc. 2. Полевая зависимость намагниченности изинговского АФМ для трех значений температур, $T / T_{\mathrm{N}}=0,11 ; 0,12 ; 0,2$, константа обмена $I_{2} / T_{\mathrm{N}}=0,6$, а константа анизотропии $D / T_{\mathrm{N}}=0,45$, где $T_{\mathrm{N}}=I_{1}+I_{2}$. Стрелки - гистерезис намагничивания при QPT, шриховыми линиями указаны точки равновесного QPT

показаны стрелками. Численные значения границ для неустойчивых решений можно найти с помощью матрицы Гессе, составленной из вторых производных свободной энергии по параметрам порядка.

\section{2. Промежуточное состояние с} плато в намагничивании наблюдает-

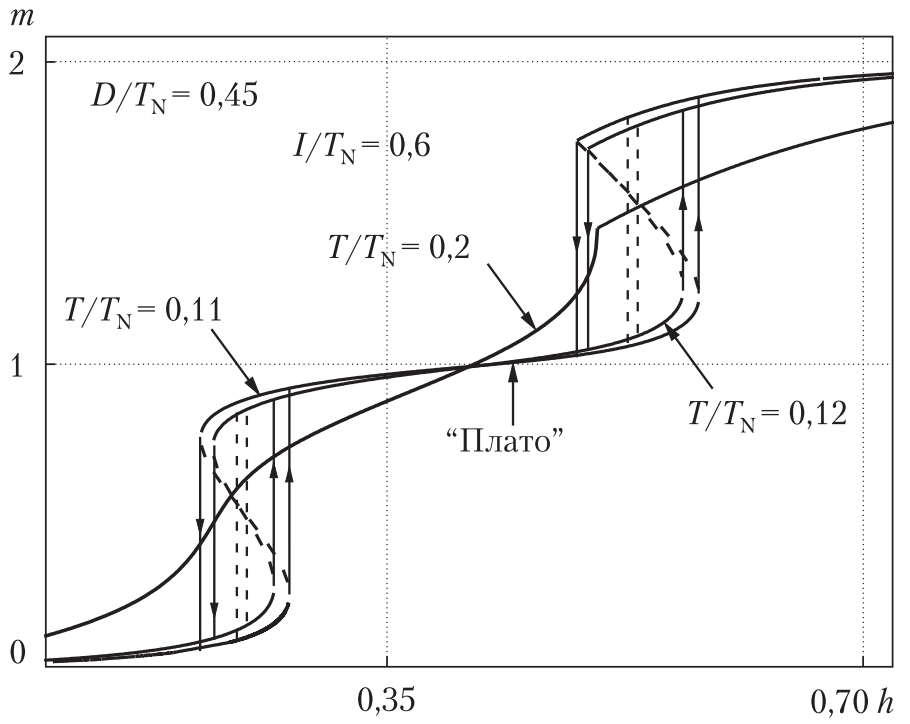
ся в интервале полей $h h_{\mathrm{VF}}^{L}<h<h_{\mathrm{VF}}^{R}$. Возникновение плато хорошо видно на рис. 2 для полевой зависимости намагниченности $m=s_{1}+s_{2}$. На рис. 1 положение QPT показано штриховой вертикальной линией. Поле QPT I находили из условия равенства энергий устойчивых решений АФМ фазы и промежуточной фазы, в которой полевая зависимость намагниченности имеет вид плато (см. рис. 2). Особенностью этого перехода, является то, что значение среднего спина $s_{1}$ первой подрешетки практически не изменяется, а величины $s_{2}$ среднего спина после перехода становится близкой нулю. При QРТ вторая подрешетка переходит в парамагнитное ван-флековское состояние, с основным состоянием $|0\rangle$ для спинов этой подрешетки.

3. Ферромагнитное состояние. В сильных полях, превышающих $h>h_{\mathrm{FM}}$, из уравнений состояния получаем ФМ решение с $s_{1}=s_{2}$. Переход в ФМ состояние также является QPT I. При этом QPT основное состояние спинов второй подрешетки изменяется с $|0\rangle$ на $|1\rangle$. Поле $h_{I I}$ этого QPT I находили из условия равенства энергий промежуточного состояния и ФМ фазы. Гистерезис в намагничивании при этом QPT лежит в интервале границ устойчивости промежуточной фазы и ФМ фазы $h_{\mathrm{FM}}<h<h_{\mathrm{VF}}^{R}$.

Таким образом, в данной работе получено, что в изинговском АФМ с легкоплоскостной анизотропией при $T \neq 0$ переход из АФМ фазы в ФМ фазу реализуется в два этапа. На первом этапе происходит QPT I в промежуточное состояние, в котором вторая подрешетка находится в ван-флековском парамагнитном состоянии. Вторым этапом является QPT I из промежуточного состояния в ФМ состояние. Оказалось, что при конечной температуре намагничивание между этими QPT имеет вид плато с практически неизменяющимся по величине модулем намагниченности.

\section{ЦИТИРОВАННАЯ ЛИТЕРАТУРА}

1. Локтев В.М., Островский В.С. Особенности статики и динамики магнитных диэлектриков с одноионной анизотропией. Физика низких температур. 1994. 20, № 10. С. 983-1016.

2. Калита В.М., Локтев В.М. Квантовые фазовые переходы и фазовая Н - Т диаграмма ван-флековского многоподрешеточного антиферромагнетика. Физика низких температур. 2006. 32, № 2. С 158-168. 
3. Розенфельд Е.В. О квантовых скачках намагниченности в магнетиках с одноионной анизотропией типа “легкая плоскость”. Письма в ЖЭТФ. 1976. 24, № 2. С. 60-64.

4. Стишов С.М. Квантовые фазовые переходы. Успехи физ наук. 2004. 174, № 8. С. 853-860.

5. Vojta M. Quantum phase transitions. Rep. Prog. Phys. 2003. 66. P. 2069-2110.

6. Koga A., Kawakami N., Sigrist M. Quantum phase transition of the $S=1$ Shastry - Sutherland model. J. Phys. Soc.Jpn. 2003. 72, № 4. P. 938-942.

7. Калита В.М., Локтев В.М. О последовательности квантовых (мета) магнитных переходов в изинговских антиферромагнетиках с одноионной анизотропией. Физика низких температур. 2005. 31 , № 7. P. $815-819$.

8. Hertz J.A. Quantum critical phenomena. Phys. Rev. B. 1976. 14. P. 1165.

9. Lavanov G.Yu., Kalita V.M., Ivanova I.M., Loktev V.M. Magnetic quantum phase transitions and entropy in Van-Vleck magnet.JMMM. 2016. 416. P. 466-474.

10. Шангина Е.Л., Долгополов В.Т. Квантовые фазовые переходы в двумерных системах. Успехи физ. наук. 2003. 173, № 8. C. 802-812.

11. Звездин А.К., Матвеев В.М., Мухин А.А., Попов А.И. Редкоземельные ионы в магнитно-упорядоченных кристаллах. Москва: Наука, 1985. 296 с.

Поступило в редакцию 30.05 .2018

\section{REFERENCES}

1. Loktev, V. M. \& Ostrovskii, V. S. (1999). The peculiarities of statics and dynamics of magnetic insulators with single-ion anisotropy. Low. Temp. Phys., 20, No. 10, pp. 983-1016 (in Russian).

2. Kalita, V. M. \& Loktev, V. M. (2006). Quantum phase transitions and phase H - T diagram of Van-Vleck multi - sublattice antiferromagnet. Low. Temp. Phys., 32, No. 2, pp. 158-168 (in Russian).

3. Rozenfeld, E. V. (1976). Quantum magnetization jump with easy - plane anisotropy. JETP. Lett., 24, No. 2, pp. 50-53.

4. Stishov, S. M. (2004). Quantum phase transitions. Phys. Sci. Uspekhi, 174, No. 8, pp. 853-860 (in Russian).

5. Vojta, M. (2003). Quantum phase transitions. Rep. Prog. Phys., 66, pp. 2069-2110.

6. Koga, A., Kawakami, N. \& Sigrist, M. (2003). Quantum phase transition of the $\mathrm{S}=1$ Shastry - Suyherland model. J. Phys. Soc. Jpn., 72. No. 4, pp. 938-942.

7. Kalita, V. M. \& Loktev, V. M. (2005). On the sequence of quantum (meta) magnetic transitions in Ising antiferromagnets with single - ion anisotropy. Low. Phys. Temp., 31, No. 7, pp. 815-819 (in Russian).

8. Hertz, J. A. (1976). Quantum critical phenomena. Phys. Rev. B., 14, pp. 1165-.

9. Lavanov, G. Yu., Kalita, V. M., Ivanova, I. M. \& Loktev, V. M. (2016). Magnetic quantum phase transitions and entropy in Van-Vleck magnet. JMMM., 416, pp. 466-474.

10. Shangina, E. L. \& Dolgopolov, V. T. (2003). Quantum phase transitions in two - dimensional systems. Phys. Sci. Uspekhi, 173, No. 8, pp. 802-812 (in Russian).

11. Zvezdin, A. K., Matveev, V. M., Mukhin, A. A. \& Popov, A. I. (1985). Rare - earth ions in magnetically ordered crystals. Moscow: Nauka (in Russian).

Received 30.05.2018

\section{Г.Ю. Лаванов}

Національний авіаційний університет, Київ

E-mail: Lavanovgennady@gmail.com

ПОДВІЙНИЙ МЕТАМАГНІТНИЙ ФАЗОВИЙ ПЕРЕХІД

У ІЗІНГІВСЬКОМУ АНТИФЕРОМАГНЕТИКУ З ЛЕГКОПЛОЩИННОЮ

ОДНОІОННОЮ АНІЗОТРОПІЕЮ ТА ЗІ СПІНОМ ІОНІВ $S=1$

Показано, що в ізінгівському АФМ зі спіном іонів $S=1$ магнітне поле при $T \neq 0$ може спонукати подвійний метамагнітний перехід у вигляді послідовності двох квантових магнітних фазових переходів першого роду (QPT I) з плато у намагнічуванні між цими переходами. При цьому перехід з антиферомагнітної фази у 
феромагнітну відбувається через проміжний стан, у якому спін підгратки, початково намагнічений проти магнітного поля, знаходиться у ван-флеківському стані.

Ключові слова: ентропія, антиферомагнетик, парамагнетик, фазові переходи.

\section{G.Yu. Lavanov}

National Aviation University, Kiev

E-mail: Lavanovgennady@gmail.com

\section{THE DOUBLE METAMAGNETIC PHASE TRANSITION \\ IN AN ISING ANTIFERROMAGNET WITH LIGHT-PLANE SINGLE-ION ANISOTROPY AND WITH ION SPIN $S=1$}

It is shown that, in an Ising AFM with ion spin $S=1$, a magnetic field at $T \neq 0$ can induce a double metamagnetic transition in the form of a sequence of two quantum first-order magnetic phase transitions (QPT I) from a plateau in the magnetization between these transitions. In this case, the transition from the antiferromagnetic phase to the ferromagnetic phase occurs through the intermediate state formation, in which the sublattice spin initially magnetized against the magnetic field is in the Van Vleck paramagnetic state.

Keywords: entropy, antiferromagnet, paramagnet, phase transitions. 